\title{
The Liver X-Receptor (Lxr) Governs Lipid Homeostasis in Zebrafish during Development
}

\author{
Amena Archer ${ }^{1}$, Satish Srinivas Kitambi ${ }^{1,2}$, Stefan L. Hallgren ${ }^{2}$, Matteo Pedrelli ${ }^{1,3}$, K. Håkan Olsén ${ }^{2}$, \\ Agneta Mode ${ }^{1}$, Jan-Åke Gustafsson ${ }^{1,4}$ \\ ${ }^{1}$ Department of Biosciences and Nutrition, Karolinska Institute, Solna, Sweden \\ ${ }^{2}$ School of Life Sciences, Södertörns University, Södertörn, Sweden \\ ${ }^{3}$ Division of Clinical Chemistry, Department of Laboratory Medicine, Karolinska Institute, Solna, Sweden \\ ${ }^{4}$ Center for Nuclear Receptors and Cell Signaling, Department of Biology and Biochemistry, University of Houston, Houston, USA \\ Email: amena.archer@ki.se
}

Received August 6, 2012; revised September 8, 2012; accepted October 9, 2012

\begin{abstract}
The liver X-receptors (lxrs) act as cholesterol sensors and participate in the regulation of lipid and cholesterol metabolism. The objective of this study was to determine the role of lxr during development using the zebrafish model. By in situ hybridization we showed distinct expression of lxr in the brain and the retina in the developing and adult zebrafish. lxr ligand activation affected the expression of genes involved in lipid metabolism in zebrafish adult brain and eye as well as in zebrafish embryos. Morpholino knock down of lxr resulted in an overall impaired lipid deposition as determined by oil red $\mathrm{O}$ staining particularly in the head and around the eyes, and to significantly elevated levels of both total and free cholesterol in the yolk of lxr morphant embryos. The expression of genes involved in lipid and cholesterol metabolism was also changed in the lxr morphants. Furthermore, alcian blue staining revealed malformation of the pharyngeal skeleton in the lxr morphant. Our data show that lxr is an important component of the regulatory network governing the lipid homeostasis during zebrafish development, which in turn may support a role of lxr for normal development of the central nervous sytem, including the retina.
\end{abstract}

Keywords: Liver X-receptor; Zebrafish; Morpholino; Lipid

\section{Introduction}

The liver X-receptors (lxrs) are ligand-activated transcription factors belonging to the nuclear receptor superfamily conserved throughout evolution [1]. In mammals there are two 1xr genes encoding the paralogues $1 \mathrm{xr} \alpha$ and $\operatorname{lxr} \beta$. $\operatorname{lxr} \alpha$ and $\operatorname{lxr} \beta$ have both overlapping and distinct expression patterns and regulate gene transcription by binding to lxr response elements in target genes. The lxrs have been identified as physiological receptors for oxidized cholesterol derivatives and are important regulators of cholesterol and lipid metabolic pathways [2]. Cholesterol is an essential component of all eukaryotic cells; excess cholesterol is associated with metabolic diseases, in particular atherosclerosis, and with various retinal disease conditions while cholesterol deficiency is associated with developmental abnormalities [3,4]. Despite the clear link between the lxrs and cholesterol metabolism and biosynthesis, lxr-deficient mice are viable and no obvious metabolic phenotype is manifested until adult age unless the mice are challenged with western type of diets [5]. However, mice deficient of both $\operatorname{lxr} \alpha$ and $\operatorname{lxr} \beta$ have abnormalities in the brain, including abberant ac- cumulation of lipid droplets [6]. Moreover, at late stage of embryogenesis and in neonates $\operatorname{lxr} \beta$ deficient mice show changes in the cerebral cortex [7]. In addition to their involvement in cholesterol metabolism, numerous data obtained in different experimental models demonstrate a role of lxrs in regulation of glucose metabolism [8] and fat storage [5].

Zebrafish, due to its small size, rapid generation time and optical transparency, is a powerful model to study vertebrate development and has also emerged as an important model for studies on human physiology and pathologies, and for screening of pharmacologic molecules. Common pathways in zebrafish and mammalian adipocyte biology have been demonstrated and diet-induced obesity in zebrafish involves pathways associated with mammalian obesity [9].

Evolutionary genomics of nuclear receptors and genome sequencing suggest the presence of only one $1 \mathrm{xr}$ gene in non-mammalian species like zebrafish and chicken [10-12]. We have recently cloned the zebrafish cDNA coding for $1 \mathrm{xr}$ and characterized its spatiotemporal expression pattern; its sequence is more homologues to 
mammalian $\operatorname{lxr} \alpha$ than $\operatorname{lxr} \beta$ while its expression pattern more resembles the profile of mammalian $\operatorname{lxr} \beta$ [10]. Moreover, zebrafish lxr is activated by the same ligands as mammalian lxrs and regulates genes in metabolic pathways in the adult zebrafish liver similarly to lxr regulatory effects in mammals. Here we have addressed the expression pattern of lxr in the brain and eye and the effects of lxr knock down on lipid homeostasis during zebrafish development.

\section{Materials and Methods}

\subsection{Zebrafish Husbandary}

All experiments were in accordance with the national guidelines for animal experiments and were approved by the Stockholm South Ethical Committee of the Swedish Animal Welfare Agency. Adult wild type zebrafish (Danio rerio) were kept under standardized conditions at $28.5^{\circ} \mathrm{C}$ with a $14 \mathrm{~h}: 10 \mathrm{~h}$ light: dark cycle. Embryos were obtained by natural mating and staged as described [13]. Embryos older than $24 \mathrm{~h}$ post fertilization (hpf) were treated with N-phenylthiourea (PTU), $0.003 \%(\mathrm{w} / \mathrm{v})$, to avoid pigmentation. Wild-type adult male fish were exposed to $4 \mu \mathrm{M}$ of the synthetic lxr agonist GW3965 (Sigma-Aldrich) or to DMSO for $18 \mathrm{~h}$. The animals were anesthetized with tricain ( $0.2 \mathrm{~g} / \mathrm{l})$ (Sigma-Aldrich), killed by decapitation and the organs were immediately dissected.

\subsection{In Situ Hybridization}

Antisense and sense probes for lxr, were used for whole mount in situ hybridization on zebrafish embryos as previously described [10]. Eyes and brain of adult fish were rinsed in phosphate buffered saline (PBS), fixed in $4 \%$ paraformaldehyde (PFA) overnight at $4{ }^{\circ} \mathrm{C}$, followed by dehydration in methanol and clearing in xylene. The tissues were embedded in paraffin, cut in $7 \mu \mathrm{m}$ transverse sections and mounted on polylysine glass slides (SigmaAldrich). Sections were dried, de-paraffinized and rehydrated in ethanol. Tissues were digested with proteinase $\mathrm{K}(10 \mathrm{mg} / \mathrm{mL})$ for $7 \mathrm{~min}$ and quickly rinsed with glycine ( $2 \mathrm{mg} / \mathrm{mL}$ ) in PBS-0.1\% Tween (PBSTw), followed by fixation in 4\% PFA for $5 \mathrm{~min}$ and then rinsed in PBSTw. Hybridization was at $55^{\circ} \mathrm{C}$ overnight. The slides were blocked in 5\% - 10\% sheep serum in PBSTw, incubated with anti-Digoxigenin-AP, Fab fragments (Roche) for 2 $\mathrm{h}$ at room temperature, washed with PBSTw and developed with NBT/BCIP in alkaline ( $\mathrm{pH}$ 9.5) staining buffer with $1 \mathrm{mM}$ Levamisol (Sigma-Aldrich).

\subsection{Morpholino Knock Down and Ligand Exposure}

The antisense morpholino targeting the exon-intron junc- tion of lxr gene (Mo lxr) and its corresponding 5-bp mismatch anti-sense morpholino (control) were obtained from Gene-Tools, LLC: Mo 1xr, 5'-GATGCTTGAGGAGAGTGTGACTCAC-3 and control, 5'-GATGGTTCAGGACAGTCTGACTGAC-3'. Morpholino oligonucleotides targeting the p53 gene were also used [14]. Wild-type embryos were injected at one cell stage and morphants from multiple clutches were analyzed. The efficiency of the morpholino was verified by PCR using the forward primer 5'-GGGACTCTCTGGGCTGTG-3' and the reverse primer 5'-AGTGACTTTGGGCCGATC3'. The control rescue injection experiment was performed using capped lxr mRNA transcribed using mMessage Machine kit (Ambion) and co-injected with the Mo lxr. For ligand treatment experiment, embryos at $1-2$ cell stage were exposed to $2 \mu \mathrm{M}$ of GW3965 or to DMSO, fresh ligand solution was added every $24 \mathrm{~h}$ and embryos were treated for 2 days and collected.

\subsection{Acridine Orange Staining and Alcian Blue Staining}

Apoptotic cell death was detected by incubating live zebrafish embryos with $10 \mu \mathrm{g} / \mathrm{ml}$ acridine orange (SigmaAldrich) in fish water in the dark for 30 min [15]. Larvae were washed with fish water and analyzed under fluorescence microscope. The acid-free cartilage alcian blue staining, was performed according to Walker et al. [16].

\subsection{Oil Red O Staining and Lipid Measurements}

Neutral lipids were stained with Oil Red O (Sigma-Aldrich); larvae were fixed in $4 \%$ PFA overnight at $4{ }^{\circ} \mathrm{C}$, washed in PBS, preincubated in 60\% 2-propanol for 30 min and stained in freshly filtered $0.3 \%$ Oil Red $\mathrm{O}$ in $60 \%$ 2-propanol for $3 \mathrm{~h}$ [17]. At $48 \mathrm{hpf}$, embryo body and yolk content were separated. Total and free cholesterol were measured as previously described [18].

\subsection{RNA Isolation, cDNA Synthesis and Quantitative PCR}

Total RNA extraction, cDNA synthesis and qPCR conditions were as previously described [10].

\subsection{Statistical Analysis}

Data are expressed as the mean \pm SEM. Statistical comparisons between groups were made by two-tailed Student's t-test or one-way Anova. Samples were considered significantly different at $\mathrm{P}<0.05$.

\section{Results}

\subsection{Expression Pattern of Lxr in the Brain and Eye of the Developing and Adult Zebrafish}

We have previously investigated the expression of $1 \mathrm{xr}$ in 
the developing and adult zebrafish; the brain and the eye showed relatively high expression levels and lxr was detected in the entire brain from $24 \mathrm{hpf}$ by whole mount in situ hybridization (WISH). However, any further characterization of the expression pattern of lxr in the eye and brain was not carried out [10].

The spatial expression profile of lxr in the developing retina was characterized by WISH. Expression of lxr was detected in the entire neural retina and especially in the lens at $36 \mathrm{hpf}$ of development (Figure 1(A)). At $72 \mathrm{hpf}$ lxr expression was confined to the retinal ganglion cell (RGC) and the retinal pigmental epithelium (RPE) layers (Figure 1(B)) in which the expression was maintained at 96 hpf (Figure 1(C)). In the retina of adult fish in situ hybridization on tissue sections was performed. Expression of lxr was detected in RGC layer, inner nuclear layer (INL) and photoreceptor layer (PRCL) (Figure 1(D)).

The expression of lxr in the brain of adult fish was analyzed by in situ hybridization on tissue sections (Figures 1(E)-(H)). The expression of lxr in the adult forebrain was scattered throughout the whole telencephalon (Figure 1(F)). In the mesencephalon, the periventricular grey zone (PGZ) of the optic tectum and the central grey zone (GC) showed staining with the lxr probe (Figure 1(G)). Staining was also detected in the caudal cerebellum $(\mathrm{CCe})$ and in the mamillary body (CM) of the inferior hypothalamic lobes (Figure 1(G)). In the rhombencephalon, $1 x r$ was detected in the caudal cerebellar lobe (LCa) and in ventral side of the rhombencephalic ventricle (RV) (Figures 1(G) and (H)). Thus, there is a profound expression of lxr in the brain and the eye/retina during zebrafish development and at the adult stage.

\subsection{GW3965 Treatment Affects the Expression of Genes Involved in Lipid Metabolism in Adult Brain and Eye as Well as in Developing Embryo in Zebrafish}

In a previous study, we showed that the zebrafish $1 x r$ is activated by the natural lxr ligand 22-R-hydroxycholesterol as well as by the synthetic lxr ligands GW3965 and T0901317 [10]. In the current study, we have chosen to use the selective and potent lxr agonist GW3965. In adult fish treated with GW3965, the expression of abca1, abcg1, scd1 and apoe in the lipid pathway was markedly up-regulated in both brain and eye whereas apoa1 and srb1 expression was not affected (Table 1). Moreover, a negative feedback regulation of $1 \mathrm{xr}$ expression in the adult zebrafish eye was observed. Thus, lxr in zebrafish is likely to be involved in the regulation of whole body lipid homeostasis including the eye and brain.

In zebrafish embryos exposed to $2 \mu \mathrm{M}$ of GW3965 from fertilization to $48 \mathrm{hpf}$, the mRNA levels of abcal, abcg1 and scd1 genes, were markedly increased when
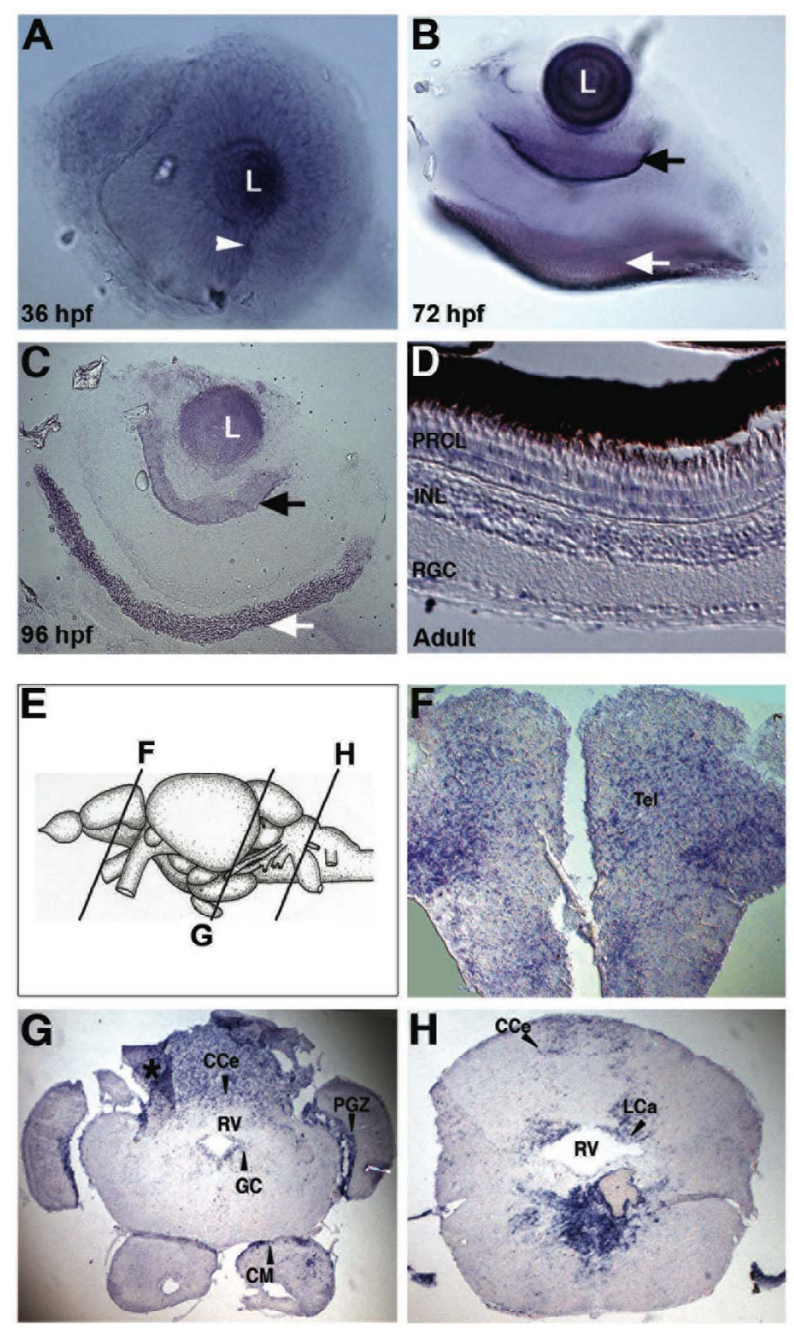

Figure 1. Expression of lxr in the brain and retina of embryo and adult zebrafish. Whole-mount in situ hybridization with anti-sense probe for lxr in the embryo zebrafish eye at 36, 72 and 96 hpf (A)-(C). The white arrowhead marks the choroid fissure, the black arrow marks retinal ganglion cell layer and the white arrow marks the retinal pigment layer. In situ hybridization was performed on adult zebrafish tissue sections in the retina (D), the telencephalon (F), mesencephalon and cerebellum (G), and rhombencephalon $(H)$. The panel $(E)$ represents the schematic location of the brain transverse sections. The asterisk marks darker area caused by tissue fold. Abbreviations: PRCL, photoreceptor layer; INL, inner nuclear layer; RGC, retinal ganglion cells; CCe, cerebellar corpus; PGZ, periventricular gray zone; GC, central gray; CM, mammaillary body; RV, rhombencephalic ventricle; LCa, caudal lobe of cerebellum.

compared to control embryos (Table 2). The cyp11a1 and cyp46a1 genes are involved in cholesterol metabolism; cyp $11 a 1$ catalyzes the first step of the steroid hormone biosynthesis [19] and cyp46a1 is brain specific and converts cholesterol into 24S-hydroxycholesterol, which, in contrast to cholesterol, can exit the brain through the blood-brain barrier [20]. GW3965 treatment had no ef- 
fect on the expression of cyp46a1 and cyp11a1 or on the expression of apoa1, apoe, $m t p$ and $s r b 1$.

\subsection{Lxr Knock Down Alters the Lipid Distribution and Homeostasis in Zebrafish Embryos during Development}

To further investigate the role of lxr for lipid homeostasis during zebrafish development, we used morpholino antisense oligonucleotides directed against the splice donor site in exon 5 of the lxr gene (Mo lxr), which encodes the DNA binding domain of the receptor (Figure 2(A)). As revealed by PCR, injection of increasing concentrations of the Mo 1xr, $10-100 \mu \mathrm{M}$, dose-dependently changed the splicing of the $1 \mathrm{xr}$ transcript (Figure 2(A)); 25 to $50 \mu \mathrm{M}$

Table 1. Gene regulatory effects of lxr ligand (GW3965) treatment in brain and eye of adult zebrafish.

\begin{tabular}{ccc}
\hline Gene & Brain & Eye \\
\hline abca1 & $6.76 \pm 0.87^{*}$ & $4.60 \pm 0.57^{*}$ \\
abcg1 & $2.99 \pm 0.22^{*}$ & $1.93 \pm 0.28^{*}$ \\
apoa1 & $0.88 \pm 0.10$ & $0.65 \pm 0.07$ \\
apoe & $3.36 \pm 0.61^{*}$ & $1.55 \pm 0.11^{*}$ \\
lxr & $1.03 \pm 0.11$ & $0.64 \pm 0.10^{*}$ \\
scd & $7.86 \pm 1.24^{*}$ & $5.39 \pm 0.06^{*}$ \\
srb1 & $1.23 \pm 0.09$ & $0.79 \pm 0.10$ \\
\hline
\end{tabular}

Adult zebrafish were treated with $4 \mu \mathrm{M}$ GW3965 (GW; n =6), or vehicle $(\mathrm{Ctrl} ; \mathrm{n}=5)$ for $18 \mathrm{~h}$. Relative mRNA levels were analyzed by qPCR. Values are expressed as fold change \pm SEM. ${ }^{*} \mathrm{P}<0.05$, vehicle vs GW3965-treated.

Table 2. Gene regulatory effects of lxr ligand (GW3965) treatment and of morpholino knockdown.

\begin{tabular}{cccc}
\hline Gene & GW3965 & MO lxr & $\begin{array}{c}\text { MO lxr + } \\
\text { MO p53 }\end{array}$ \\
\hline abca1 & $3.75 \pm 0.13^{*}$ & $0.64 \pm 0.11^{*}$ & $0.54 \pm 0.06$ \\
abcg1 & $4.23 \pm 0.42^{*}$ & $0.50 \pm 0.04^{*}$ & $0.47 \pm 0.04$ \\
apoa1 & $0.61 \pm 0.08$ & $1.35 \pm 0.21$ & $1.60 \pm 0.21$ \\
apoe & $1.10 \pm 0.16$ & $1.25 \pm 0.10$ & $1.21 \pm 0.08$ \\
cyp11a1 & $2.20 \pm 0.18$ & $1.52 \pm 0.15^{*}$ & $1.19 \pm 0.12$ \\
cyp46a1 & $1.20 \pm 0.13$ & $1.37 \pm 0.08^{*}$ & $1.17 \pm 0.06$ \\
mtp & $1.31 \pm 0.23$ & $1.51 \pm 0.22$ & $2.13 \pm 0.04^{*}$ \\
scd & $1.75 \pm 0.20^{*}$ & $0.85 \pm 0.05$ & $1.01 \pm 0.06$ \\
srb1 & $1.02 \pm 0.16$ & $1.23 \pm 0.06$ & $1.43 \pm 0.06$ \\
\hline
\end{tabular}

Relative mRNA levels were analyzed by qPCR in 4 independent pools of embryos at 48 hpf. Embryos were exposed to vehicle or $2 \mu \mathrm{M}$ GW3965 from 1 - 2 cell stage till $48 \mathrm{hpf}$ or injected with contol morpholino or Mo lxr together or not with Mo p53. Values are expressed as fold change \pm SEM. ${ }^{*} \mathrm{P}$ $<0.05$, vehicle vs GW3965-treated embryos, control-mismatch vs Mo lxr-injected embryos and Mo lxr vs Mo 1xr + Mo p53. were used in subsequent experiments. Cell death is a natural process occurring during development, however, the generally reduced size of the head and eyes and the opacity of these structures in the 1xr morphants (Figures 2(B) and (C)) led us to analyze cell apoptosis by acridine orange staining [15]. At $26 \mathrm{hpf}$ the fluorescence signal was particularly high in the midbrain, hindbrain and eye of the lxr morphants (Figures 2(E) and (F)). A high cell death rate and small eyes and brain are frequently associated with non-specific effects of morpholinos on the p53 pathway [14] and by co-injection of Mo p53 the cell death rate in Mo lxr embryos was partially decreased (Figures 2(D) and (G)) suggesting non-specific effects also of the Mo lxr, however, the head and the eyes were still smaller than in the control embryos. The p21 gene is tightly controlled by p53 [14] and served as a control in these experiments (Figure 2(N)).

To determine whether the lipid homeostasis was perturbed in lxr morphants, we performed staining of neutral lipids with oil Red O (ORO). The staining revealed a dramatic decrease of lipid deposit in the entire body in $95 \%$ of the lxr morphants, in particular in the head and around the eyes at $24 \mathrm{hpf}$ (Figures 2(H) and (I)). Similar results were observed at $48 \mathrm{hpf}$ (data not shown). The vasculature appeared normally formed in the lxr morphants as revealed by similar expression of the fli1: EGFP transgene in the trunk of control and morphants, demonstrating that the decrease of lipid deposit in the morphants was not due to failure in angiogenesis (Supplementary data). The lipid distribution between the embryo body and the yolk was further investigated by measuring the content of cholesterol in the two compartments at $48 \mathrm{hpf}$ (Figure 2(O)); a significant increase of both total and free cholesterol was observed in the yolk of lxr morphants compared to the control. Next we analyzed the relative mRNA levels of genes in lipid metabolic pathways in the Mo lxr and control embryos at $48 \mathrm{hpf}$ (Table 2). The $a b c a 1$ and $a b c g 1$ genes were significantly downregulated in the lxr morphants; the cyp46a1 and cyp11a1 genes were upregulated whereas the apoa1, apoe, scd and $s r b 1$ genes were not significantly affected. The effects of Mo lxr on lipid deposition and expression of genes in the lipid pathway were not modified by co-injection of the Mo p53 (Figure 2(J) and Table 2) indicating specific effects of the Mo lxr on the lipid pathway.

To further verify the specificity of the Mo lxr effect on lipid homeostasis, we exposed lxr morphants to GW3965 (Figure 3(A)); the GW3965-induced expression of $a b c a 1$ and $a b c g 1$ was attenuated in Mo lxr injected embryos compared to control embryos. Moreover, after coinjection of lxr mRNA with Mo lxr, we observed an improvement of the ORO staining in a dose-dependent manner in embryos at $72 \mathrm{hpf}$ and the expression of abca1 was significantly rescued (Figure 3(B)). 


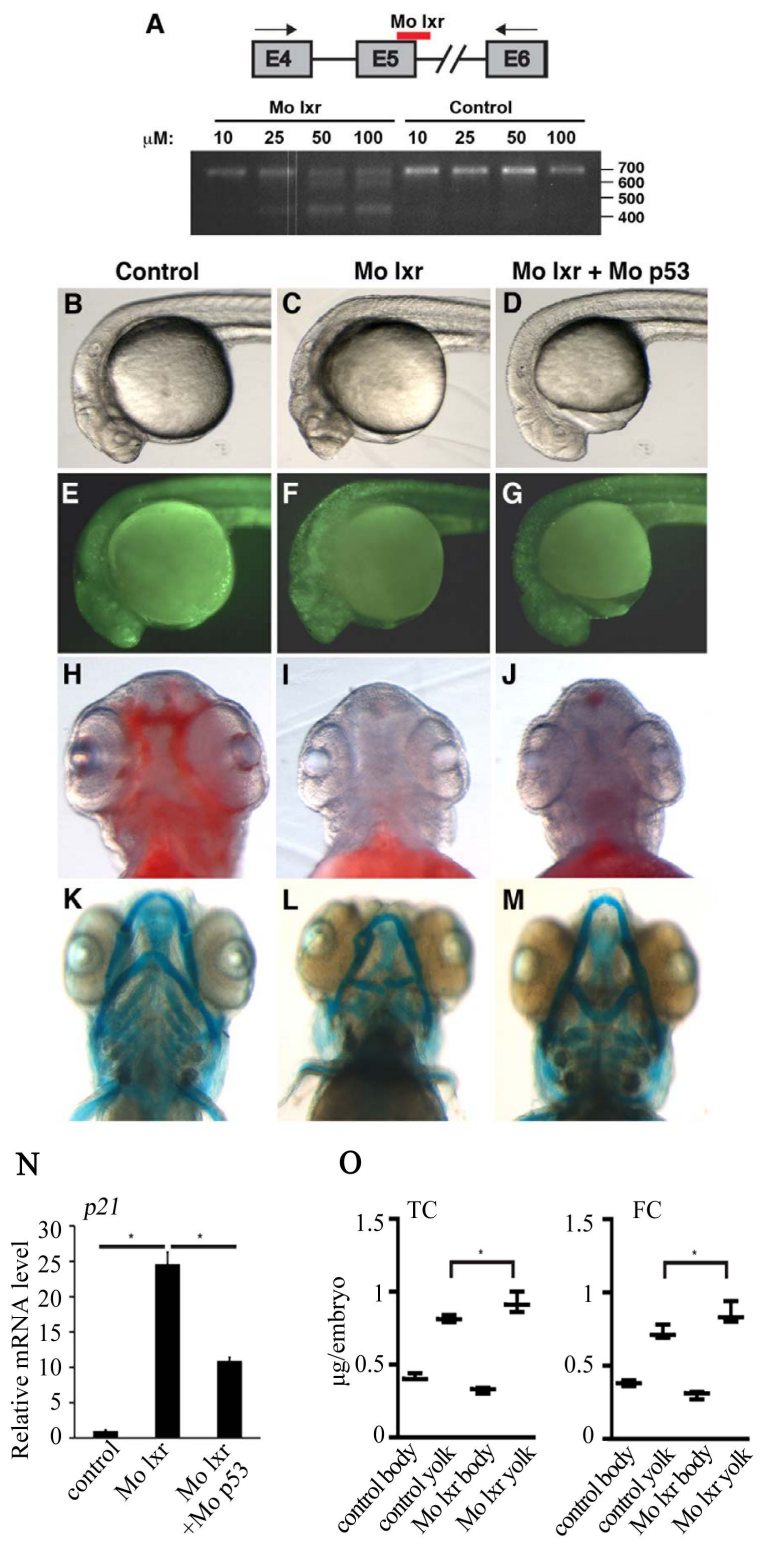

Figure 2. Phenotype of Ixr morphant embryos. (A) Schematic view of lxr exon 4, 5 and 6 with the target site of the Ixr exon 5 morpholino oligonucleotide. Arrows indicate the location of primers used for RT-PCR (lower panel). Total RNA was isolated from a pool of 20 embryos, at $24 \mathrm{hpf}$, injected with different concentrations $(10-100 \mu \mathrm{M})$ of $\mathbf{l x r}$ morpholino (Mo lxr) or mismatch morpholino (Control). (B)-(D) Bright field view and, (E)-(G) acridine orange staining for cell death at $26 \mathrm{hpf},(\mathrm{H})-(\mathrm{J})$ whole-mount oil red $\mathrm{O}$ staining (ORO) for neutral lipids at $72 \mathrm{hpf}$ and (K)-(M) whole-mount alcian blue staining for cartilage at $5 \mathrm{dpf}$ of embryos injected with morpholino lxr (Mo lxr), morpholino lxr and morpholino p53 (Mo lxr + Mo p53) or with mismatch morpholino (Control). (N) Relative mRNA levels of p21 in 48 hpf embryos. (O) Lipid levels ( $\mu \mathrm{g} / \mathrm{embryo}$ ) in the body and the yolk of Mo lxr and control embryos, total cholesterol (TC) and free cholesterol (FC) at 48 hpf. Values represent the mean \pm SEM of 3 - 4 groups $(n=35-65)$. Significant differences are indicated with asterisks, ${ }^{*} \mathbf{P}<0.05$.
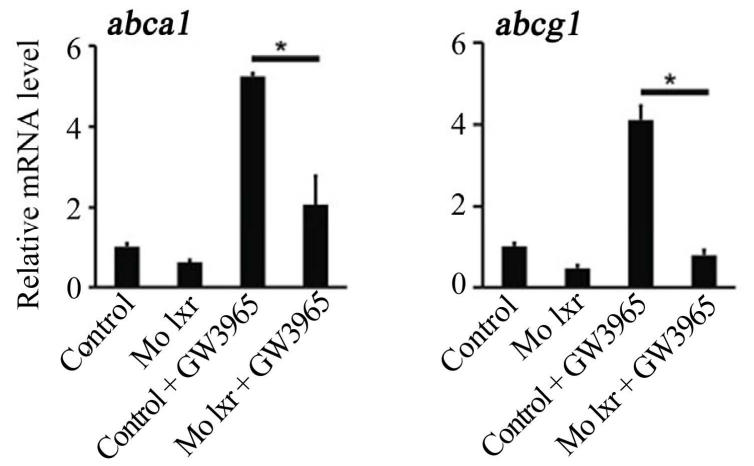

(A)
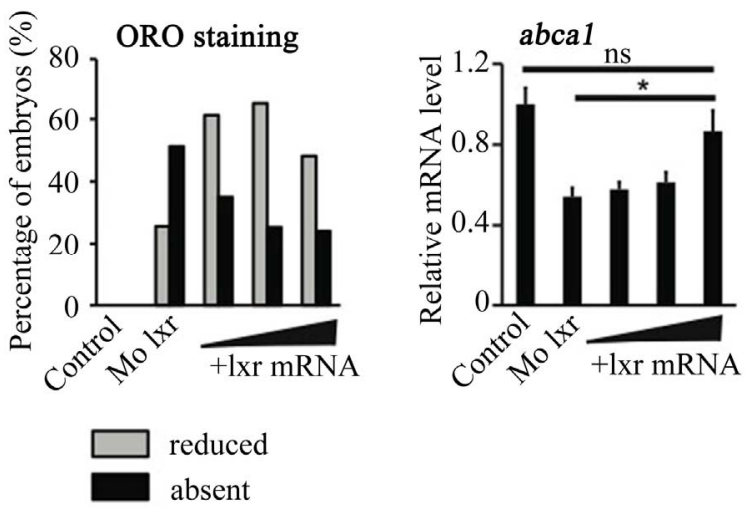

(B)

Figure 3. Effect of the GW3965 ligand and lxr mRNA injection in Mo lxr embryos. (A) Relative mRNA levels of abca1 and abcg1 in 48 hpf embryos injected with Mo lxr or mismatch morpholino (Control) and treated with vehicle or 2 mM GW3965. (B) Percentage of embryos stained by whole-mount oil red $O$ (ORO) at 72 hpf; embryos injected with mismatch morpholino, Mo lxr or with Mo lxr and increasing doses of Ixr mRNA $(25,50,250 \mathrm{pg})(\mathrm{n}=80-100)$ and relative mRNA levels of abca1. Values represent the mean \pm SEM of 4 groups $(n=20)$. Significant differences are indicated with asterisks, ${ }^{*} \mathbf{P}<\mathbf{0 . 0 5}$.

Given the connection between general metabolism and bone development we performed alcian blue staining to visualize the pharyngeal skeleton (Figures 2(K)-(M)). The severity of the jaw cartilage malformations in the lxr morphant larvae varied somewhat but consistently, the Meckel's cartilage was abnormally shaped with a more circular structure, also the ceratohyal arch was miscurved and misprojected (Figure 2(L)). Head cartilage malformations were observed also when Mo p53 was co-injected with Mo lxr (Figure 2(M)), this applied in particular to the ceratohyal arch.

\section{Discussion}

Lxr promotes cellular cholesterol efflux and activates fatty acid synthesis $[21,22]$. We and others have previously shown that zebrafish lxr responds to lxr ligands and 
regulates the expression of genes in lipid metabolic pathways in the zebrafish liver $[10,23]$. The observed lxr morphant phenotype with markedly reduced yolk utilization, impaired lipid disposition in the entire body and reduced expression of lipid metabolic genes confirm an evolutionarily conserved role of $1 x r$ in lipid homeostasis from teleosts to mammalian species. Zebrafish embryos and larvae with the nutrient supply coming solely from the yolk represent a closed nutritional system compared to mammals. Neutral lipids from the yolk can be stored as deposits in the larval body and then released during development when required [24,25]. Ultrastructural evidence for very low density lipoprotein (VLDL) synthesis in the vitelline syncytium implies packaging of complex lipids for delivery to developing tissues in the rainbow trout [26]. In mammals, it has been suggested that a process of VLDL-like particle formation and secretion to the fetus could occur both from the yolk sac and the placenta $[27,28]$. Furthermore, the production of large VLDL particles in the liver is stimulated by lxr activation [29]. Thus, lxr-dependent production and/or secretion of VLDL-like particles from the yolk to the developing zebrafish embryo can be inferred. Moreover, the lipid transporters $\mathrm{ABCA} 1$ and $\mathrm{ABCG} 1$ appear to be involved in the delivery of cholesterol from the placenta to the fetal circulation in the human [30]. In the lxr morphants the overall lipid disposition was severely affected as shown by ORO staining, and abcal and abcg1 expression was dramatically decreased. The abcal gene appears to be a prominent target gene in the zebrafish that was also markedly induced by lxr-ligand activation. Our results suggest that the impaired lipid distribution observed in the lxr morphants could be linked to the down regulation of key lipid transporters such as Abcal and $A b c g 1$. All together, accumulating data suggest a major role of lxr in the regulation of lipid transport to the developing embryo, from the yolk in oviparous animals or via the placenta in mammals.

We observed significantly higher levels of both total and free cholesterol in the yolk of lxr morphants compared to the control, which could indicate a deficiency in the developing embryo. A number of genetic defects affecting specific steps in cholesterol synthesis, handling or storage are known to lie behind cartilage and skeletal malformations. For example, cholesterol deficiency in the inherited Smith-Lemli-Opitz syndrome, is caused by mutation in the 7-dehydrocholesterol reductase gene, which leads to dysmorphic craniofacial features and brain defects $[3,4]$. Indeed, lxr has been shown to regulate cholesterol homeostasis in the central nervous system (CNS) [31]. It has been described that $\operatorname{lxr} \beta$ knock out mice at late stage of embryogenesis have a smaller brain with a reduction of neurons in the superficial cortical layer [7] and that in $\operatorname{lxr} \alpha \beta$ deficient mice there is an un- balance between neurons and astrocytes in the ventral midbrain [32]. Moreover, a recent study on the huntingtin protein, a nuclear protein that binds to a number of transcription factors, shows that this protein regulates lxr-mediated gene transcription and the huntingtin morpholino knock down zebrafish shows reduced expression of abcg1 [33]. The cartilage phenotype in the huntingtin morphant was shown to be partially rescued by lxr agonist treatment. Using whole-mount alcian blue staining, we observed a very high frequency of craniofacial cartilage defects in the lxr morphants similar to those described for the huntingtin morpholino knock down zebrafish. Consequently, it is plausible that deficiency of specific lipids [17,34], e.g. cholesterol, contributed to the cartilage phenotype of the lxr morphant.

The morpholino knock down technology is a very powerful tool that has allowed important steps for the comprehension of gene function during vertebrate development. However, there are general disadvantages associated with this technology including unspecific effects and problems with efficiency of rescuing. High levels of cell death and developmental delay are frequently reported upon injection of morpholinos. The time of onset of translation may be very important for developmental genes and the co-injection at early stage of development of mRNA and morpholino to rescue the morpholino effect may affect developmental processes and/ or reduce the efficiency of the rescue. In this study, we observed an impaired lipid metabolism and high level of cell death. While the high level of cell death was significantly suppressed when co-injecting Mo p53, the lipid metabolism impairment was not affected indicating that the lipid phenotype constitutes a specific effect. The incomplete rescue of the lipid phenotype following coinjection of lxr mRNA could possibly be due to lack of accuracy in targeting the appropriate time point and geographic area for lxr expression during zebrafish development. However, given the involvement of $1 \mathrm{xr}$ in cholesterol and lipid metabolism/traffic and the crucial role of lipid supply from the yolk for the zebrafish embryo development this phenotype was expected in the lxr morphant. To take full advantage of the zebrafish model in exploring the role of $1 x r$ in development the use of additional techniques to modulate lxr expression in zebrafish such as ENU mutant [35] or zinc-finger knock out [36] models is required.

Taken together, our results show that the zebrafish lxr is an important component of the regulatory network governing the lipid metabolism pathway during zebrafish development. This is concordant with the importance of lipids in general and cholesterol in particular for the formation and maintenance of the nervous system. Possibly, the zebrafish model is more susceptible than the mouse model to impairment of lipid metabolism and could be 
useful to highlight new aspects of the role of $1 \mathrm{xr}$ for the development and functioning of the eye and the brain.

\section{Acknowledgements}

This study was supported by grants from the Swedish Research Council (1213-45762) and Karolinska Institutet. We thank Dr. Monika Andersson-Lendahl and Kent Ivarsen for zebrafish husbandary, Christina Thulin-Andersson and Charlotte Six for their technical help and Dr. Arindam Majumdar for valuable discussions and comments on the manuscript.

\section{REFERENCES}

[1] S. Bertrand, B. Thisse, R. Tavares, et al., "Unexpected Novel Relational Links Uncovered by Extensive Developmental Profiling of Nuclear Receptor Expression," PLoS Genetics, Vol. 3, No. 11, 2007, p. e188. doi:10.1371/journal.pgen.0030188

[2] B. A. Janowski, M. J. Grogan, S. A. Jones, et al., "Structural Requirements of Ligands for the Oxysterol Liver X Receptors Lxralpha and Lxrbeta," Proceedings of the National Acadamy of Sciences of the USA, 5 January 1999, pp. 266-271. doi:10.1073/pnas.96.1.266

[3] G. Hoffmann, K. M. Gibson, I. K. Brandt, P. I. Bader, R. S. Wappner and L. Sweetman, "Mevalonic Aciduria-An Inborn Error of Cholesterol and Nonsterol Isoprene Biosynthesis," New England Journal of Medicine, Vol. 314, No. 25. 1986, pp. 1610-1614. doi:10.1056/NEJM198606193142504

[4] D. W. Smith, L. Lemli and J. M. Opitz, "A Newly Recognized Syndrome of Multiple Congenital Anomalies," The Journal of Pediatrics, Vol. 64, 1964, pp. 210-217. doi:10.1016/S0022-3476(64)80264-X

[5] N. Y. Kalaany, K. C. Gauthier, A. M. Zavacki, et al., "Lxrs Regulate the Balance between Fat Storage and Oxidation," Cell Metabolism, Vol. 1, No. 4, 2005, pp. 231-244. doi:10.1016/j.cmet.2005.03.001

[6] L. Wang, G. U. Schuster, K. Hultenby, Q. Zhang, S. Andersson and J. A. Gustafsson, "Liver X Receptors in the Central Nervous System: From Lipid Homeostasis to Neuronal Degeneration," Proceedings of the National Acadamy of Sciences of the USA, 15 October 2002, pp. 13878-13883. doi:10.1073/pnas.172510899

[7] X. Fan, H. J. Kim, D. Bouton, M. Warner and J. A. Gustafsson, "Expression of Liver X Receptor Beta Is Essential for Formation of Superficial Cortical Layers and Migration of Later-Born Neurons," Proceedings of the National Acadamy of Sciences of the USA, 9 September 2008, pp. 13445-13450. doi:10.1073/pnas.0806974105

[8] B. A. Laffitte, L. C. Chao, J. Li, et al., "Activation of Liver X Receptor Improves Glucose Tolerance through Coordinate Regulation of Glucose Metabolism in Liver and Adipose Tissue," Proceedings of the National Acadamy of Sciences of the USA, 29 April 2003, pp. 5419-5424. doi:10.1073/pnas.0830671100

[9] E. J. Flynn, C. M. Trent and J. F. Rawls, “Ontogeny and Nutritional Control of Adipogenesis in Zebrafish (Danio rerio)," Journal of LIPID Research, Vol. 50, No. 8, 2009 , pp. 1641-1652. doi:10.1194/jlr.M800590-JLR200

[10] A. Archer, G. Lauter, G. Hauptmann, A. Mode and J. A. Gustafsson, "Transcriptional Activity and Developmental Expression of Liver X-Receptor (Lxr) in Zebrafish," Developmental Dynamics, Vol. 237, No. 4, 2008, pp. 1090-1098. doi:10.1002/dvdy.21476

[11] S. Bertrand, F. G. Brunet, H. Escriva, G. Parmentier, V. Laudet and M. Robinson-Rechavi, "Evolutionary Genomics of Nuclear Receptors: From Twenty-Five Ancestral Genes to Derived Endocrine Systems," Molecular Biology and Evolution, Vol. 21, No. 10, 2004, pp. 19231937. doi: $10.1093 / \mathrm{molbev} / \mathrm{msh} 200$

[12] E. J. Reschly, N. Ai, W. J. Welsh, S. Ekins, L. R. Hagey and M. D. Krasowski, "Ligand Specificity and Evolution of Liver X-Receptors," The Journal of Steroid Biochemistry and Molecular Biology, Vol. 110, No. 1-2, 2008 , pp. 83-94. doi:10.1016/j.jsbmb.2008.02.007

[13] C. B. Kimmel, W. W. Ballard, S. R. Kimmel, B. Ullmann and T. F. Schilling, "Stages of Embryonic Development of the Zebrafish," Developmental Dynamics, Vol. 203, No. 3, 1995, pp. 253-310. doi:10.1002/aja.1002030302

[14] U. Langheinrich, E. Hennen, G. Stott and G. Vacun, “Zebrafish as a Model Organism for the Identification and Characterization of Drugs and Genes Affecting p53 Signaling," Current Biology, Vol. 12, No. 23, 2002, pp. 2023-2028. doi:10.1016/S0960-9822(02)01319-2

[15] B. Tucker and M. Lardelli, "A Rapid Apoptosis Assay Measuring Relative Acridine Orange Fluorescence in Zebrafish Embryos," Zebrafish, Vol. 4, No. 2, 2007, pp. 113-116. doi:10.1089/zeb.2007.0508

[16] M. B. Walker and C. B. Kimmel, "A Two-Color Acid-Free Cartilage and Bone Stain for Zebrafish Larvae," Biotechnic and Histochemistry, Vol. 82, No. 1, 2007, pp. 23-28. doi:10.1080/10520290701333558

[17] K. Schlombs, T. Wagner and J. Scheel, "Site-1 Protease Is Required for Cartilage Development in Zebrafish," Proceedings of the National Acadamy of Sciences of the USA, 2003, pp. 14024-14029.

doi:10.1073/pnas.2331794100

[18] M. Korach-Andre, A. Archer, C. Gabbi, et al., "Liver X Receptors Regulate de Novo Lipogenesis in a Tissue-Specific Manner in C57BL/6 Female Mice," American Journal of Physiology Endocrinology and Metabolism, Vol. 301, No. 1, 2011, pp. E210-E222. doi:10.1152/ajpendo.00541.2010

[19] H. J. Hsu, N. C. Hsu, M. C. Hu and B. C. Chung, "Steroidogenesis in Zebrafish and Mouse Models," Molecular and Cellular Endocrinology, Vol. 248, No. 1-2, 2006, pp. 160-163. doi:10.1016/j.mce.2005.10.011

[20] D. Lutjohann, O. Breuer, G. Ahlborg, et al., "Cholesterol Homeostasis in Human Brain: Evidence for an Age-Dependent Flux of 24S-Hydroxycholesterol from the Brain into the Circulation," Proceedings of the National Acadamy of Sciences of the USA, 1996, pp. 9799-9804. doi:10.1073/pnas.93.18.9799

[21] D. J. Peet, B. A. Janowski and D. J. Mangelsdorf, "The Lxrs: A New Class of Oxysterol Receptors," Current Opinion in Genetics \& Development, Vol. 8, No. 5, 1998, 
pp. 571-575. doi:10.1016/S0959-437X(98)80013-0

[22] J. R. Schultz, H. Tu, A. Luk, et al., "Role of Lxrs in Control of Lipogenesis," Genes and Development, Vol. 14, No. 22, 2000, pp. 2831-2838. doi:10.1101/gad. 850400

[23] H. Sukardi, X. Zhang, E. Y. Lui, et al., "Liver X-Receptor Agonist T0901317 Induced Liver Perturbation in Zebrafish: Histological, Gene Set Enrichment and Expression Analyses," Biochimica et Biophysica Acta (BBA)—General Subjects, Vol. 1820, No. 1, 2011, pp. 33-43. doi:10.1016/j.bbagen.2011.10.009

[24] D. R. Tocher, A. J. Fraser, J. R. Sargent and J. C. Gamble, "Lipid Class Composition during Embryonic and Early Larval Development in Atlantic Herring," Lipids, Vol. 20, No. 2, 1985, pp. 84-89. doi:10.1007/BF02534210

[25] D. R. Tocher, A. J. Fraser, J. R. Sargent and J. C. Gamble, "Fatty Acid Composition of Phospholipids and Neutral Lipids during Embryonic and Early Larval Development in Atlantic Herring," Lipids, Vol. 20, No. 2, 1985, pp. 69-74. doi:10.1007/BF02534210

[26] J. M. Vernier and M. F. Sire, "Lipoprotéines de très Basse Densité et Glycogène Dans le Syncytium Vitellin, L'Épithélium Intestinal et le Foie, Aux Stades Précoces du Developement Embryonnaire Chez la Truite Arc-enCiel," Biologie cellulaire, Vol. 29, 1977, pp. 45-54.

[27] E. M. Madsen, M. L. Lindegaard, C. B. Andersen, P. Damm and L. B. Nielsen, "Human Placenta Secretes Apolipoprotein B-100-Containing Lipoproteins," The Journal of Biological Chemistry, Vol. 279, No. 53, 2004, pp. 55271-55276. doi:10.1074/jbc.M411404200

[28] Y. Terasawa, S. J. Cases, J. S. Wong, et al., "Apolipoprotein B-Related Gene Expression and Ultrastructural Characteristics of Lipoprotein Secretion in Mouse Yolk Sac during Embryonic Development," Journal of Lipid Research, Vol. 40, No. 11, 1999, pp. 1967-1977.

[29] A. Grefhorst, B. M. Elzinga, P. J. Voshol, et al., "Stimulation of Lipogenesis by Pharmacological Activation of the Liver X Receptor Leads to Production of Large, Triglyceride-Rich Very Low Density Lipoprotein Parti- cles," Journal of Biological Chemistry, Vol. 277, No. 37, 2002, pp. 34182-34190. doi:10.1074/jbc.M204887200

[30] J. Stefulj, U. Panzenboeck, T. Becker, et al., "Human Endothelial Cells of the Placental Barrier Efficiently Deliver Cholesterol to the Fetal Circulation via ABCA1 and ABCG1," Circulation Research, Vol. 104, No. 5, 2009, pp. 600-608. doi:10.1161/CIRCRESAHA.108.185066

[31] J. J. Repa, H. Li, T. C. Frank-Cannon, et al., "Liver X-Receptor Activation Enhances Cholesterol Loss from the Brain, Decreases Neuroinflammation, and Increases Survival of the NPC1 Mouse," The Journal of Neuroscience, Vol. 27, No. 52, 2007, pp. 14470-14480. doi:10.1523/JNEUROSCI.4823-07.2007

[32] P. Sacchetti, K. M. Sousa, A. C. Hall, et al., "Liver X-Receptors and Oxysterols Promote Ventral Midbrain Neurogenesis in Vivo and in Human Embryonic Stem Cells," Cell Stem Cell, Vol. 5, No. 4, 2009, pp. 409-419. doi:10.1016/j.stem.2009.08.019

[33] M. Futter, H. Diekmann, E. Schoenmakers, O. Sadiq, K. Chatterjee and D. C. Rubinsztein, "Wild-Type but Not Mutant Huntingtin Modulates the Transcriptional Activity of Liver X-Receptors," Journal of Medical Genetics, Vol. 46, No. 7, 2009, pp. 438-446. doi:10.1136/jmg.2009.066399

[34] S. Wu and F. De Luca, "Role of Cholesterol in the Regulation of Growth Plate Chondrogenesis and Longitudinal Bone Growth," Journal of Biological Chemistry, Vol. 279, No. 6, 2004, pp. 4642-4647. doi:10.1136/jmg.2009.066399

[35] P. Haffter, M. Granato, M. Brand, et al., "The Identification of Genes with Unique and Essential Functions in the Development of the Zebrafish, Danio Rerio," Development, Vol. 123, 1996, pp. 1-36. doi:10.1074/jbc.M305518200

[36] X. Meng, M. B. Noyes, L. J. Zhu, N. D. Lawson and S. A. Wolfe, "Targeted Gene Inactivation in Zebrafish Using Engineered Zinc-Finger Nucleases," Nature Biotechnology, Vol. 26, No. 6, 2008, pp. 695-701. doi: $10.1038 / \mathrm{nbt} 1398$ 